\title{
Management of Patients with Long OT Syndrome
}

\author{
Yongkeun Cho, MD \\ Department of Internal Medicine, Kyungpook National Univeristy Hospital, Daegu, Korea
}

Long QT syndrome (LOTS) is a rare cardiac channelopathy associated with syncope and sudden death due to torsades de pointes and ventricular fibrillation. Syncope and sudden death are frequently associated with physical and emotional stress. Management of patients with LQTS consists of life-style modification, B-blockers, left cardiac sympathetic denervation (LCSD), and implantable cardioverterdefibrillator (ICD) implantation. Prohibition of competitive exercise and avoidance of QT-prolonging drugs are important issues in life-style modification. Although B-blockers are the primary treatment modality for patients with LOTS, these drugs are not completely effective in some patients. Lifelong ICD implantation in young and active patients is associated with significant complications. LCSD is a relatively simple and highly effective surgical procedure. However, LCSD is rarely used. (Korean Circ J 2016;46(6):747-752)

KEY WORDS: Long OT syndrome; Adrenergic beta-blocker; Sympathectomy; Defibrillators, implantable.

\section{Introduction}

Long OT syndrome (LQTS) is one of the most important causes of syncope and sudden death in the young. ${ }^{12)}$ Effective therapies to prevent syncope and sudden death in such patients are readily available nowadays. ${ }^{3)}$ As syncope and sudden death are due to torsades de pointes and ventricular fibrillation, management of LOTS patients means prevention of development of tachyarrhythmia (Fig. 1). Management modalities consist of life-style modification, B-blockers, left cardiac sympathetic denervation (LCSD), and implantable cardioverter-defibrillator (ICD) implantation. Genotypephenotype correlations are also important in the management of these patients.

Received: February 11, 2016

Revision Received: March 19, 2016

Accepted: March 22, 2016

Correspondence: Yongkeun Cho, MD, Department of Internal Medicine, Kyungpook National University Hospital, 130 Dongdeok-ro, Jung-gu, Daegu 41944, Korea

Tel: 82-53-420-5528, Fax: 82-53-426-2046

E-mail:choyk@knu.ac.kr

- The authors have no financial conflicts of interest.

This is an Open Access article distributed under the terms of the Creative Commons Attribution Non-Commercial License (http://creativecommons. org/licenses/by-nc/3.0) which permits unrestricted non-commercial use, distribution, and reproduction in any medium, provided the original work is properly cited.

\section{Life-Style Modification}

Life-style modification such as avoidance of strenuous exercise, especially swimming or water sports in LOT1 patients, reduction in exposure to abrupt loud noises (alarm clock, phone ringing, etc) in LOT2 patients, and avoidance of drugs that prolong the OT interval in all LOTS patients, should be routinely performed. ${ }^{3-5)}$

According to the 36 th Bethesda Conference guidelines, symptomatic patients with LOTS should be limited to Class $1 \mathrm{~A}$ sports such as brisk walking, hiking, golf, bowling, and skating. ${ }^{6}$ While asymptomatic genotype-positive/phenotype-negative patients can be allowed to play competitive sports except for swimming in LQT1 as the risk of sudden death is not zero. $\left.{ }^{6}\right)$ However, according to the more restrictive European Society of Cardiology guidelines, all LOTS patients should be disqualified from all competitive sports based on the OTc cutoff (>440 ms in males, $>460 \mathrm{~ms}$ in females), whether symptomatic or asymptomatic.7)

Currently, there is a debate regarding participation in competitive sports among experts, especially in genotype-positive/phenotypenegative patients. Some experts think that these guideline-based recommendations for disqualification are excessive and patients may participate in competitive sports safely.8) Recently revised recommendations allow some consideration of competitive sports after ensuring that effective treatment and appropriate precautionary measures such as automatic external defibrillators and personnel trained in basic life support are available, especially 


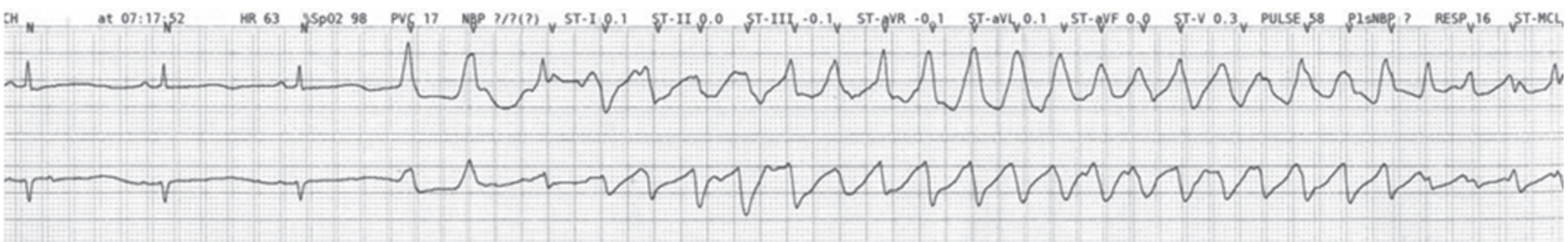

Fig. 1. Development of torsades de pointes in a young lady with long QT syndrome.

among asymptomatic genotype-positive/phenotype-negative patients. ${ }^{899)}$ Many patients with ICDs including those with LOTS can engage in vigorous competitive sports without significant morbidity and mortality. ${ }^{10)}$ Recommendation for genotype-positive/ phenotype-negative patients is also influenced by physicians' personal exercise habits; less-active physicians are more likely to restrict exercise. ${ }^{11)}$ Risk of cardiac events during sexual activity seems to be extremely low. ${ }^{12)}$

The development of cardiac events in LOT2 patients is associated with emotional stress and sudden exposure to auditory stimuli, such as noise from telephones, alarm clocks, and crying babies; hence, these sounds should be avoided.4.13) As the OTc interval in LOT2 patients is also dependent on the serum potassium level, the potassium level should be maintained within normal limits. Special care is needed in postpartum women as the risk of cardiac events is increased during the 9-month postpartum period, especially in LOT2. ${ }^{414)}$ The 9-month postpartum period is associated with a 2.7fold increased risk of cardiac events and a 4.1-fold increased risk of life-threatening events when compared with the preconception period. As sleep disturbance and emotional stress in mothers of nursing infants may trigger cardiac events, it is recommended that other family members should take care of infants at nighttime without disturbing the mothers. ${ }^{15)}$

Some patients with LOTS also suffer from asthma. Asthma comorbidity in LOTS patients is associated with increased risk of cardiac events, which is diminished after initiation of B-blocker therapy. ${ }^{16)} \beta_{2}$-agonist therapy in asthmatic LOTS patients increases the risk of cardiac events. ${ }^{17)}$ Asthma in LOTS patients may mean a more severe gene mutation since KVLOT1 seems to be essential for chloride secretion in tracheal epithelial cells. ${ }^{18)}$ Intravenous aminophylline and salbutamol should be avoided and inhaled anticholinergic medication, corticosteroids, intravenous magnesium, and leukotriene receptor antagonists may be considered as alternatives. ${ }^{19)}$

All patients should avoid exposure to OT prolonging drugs that block the $I_{k r}$ current. The list of such drugs is available at www. crediblemeds.org and it should be given to the patient and/or family members. Herbal medicines including liquorice ${ }^{20)}$ and grapefruit ${ }^{21)}$ should be avoided. The effect of oral contraceptives seems to be neutral. ${ }^{22)}$ LOTS patients treated with attention deficit/hyperactivity disorder medications showed increased risk of cardiac events, particularly syncope, and this risk is increased in male patients. ${ }^{23)}$

\section{B-Blockers}

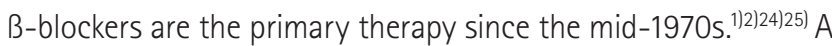
recent study showed that $B$-blockers were still the first-line therapy in 76\% of European centers. ${ }^{26)}$ B-blockers were associated with a significant reduction in cardiac events in LOTS probands and in the affected family members; $0.97 \pm 1.42$ to $0.31 \pm 0.86$ and $0.26 \pm 0.84$ to $0.15 \pm 0.69$ events per year, respectively. ${ }^{24)}$ Unfortunately, cardiac events continue to occur while patients are taking the prescribed B-blockers, especially in symptomatic patients; $32 \%$ of symptomatic patients will have a cardiac event over 5 years, and 14\% of patients with a prior cardiac arrest will have a recurrence within 5 years. ${ }^{24)}$

As B-blockers are extremely effective in LOT1, B-blocker noncompliance and use of OT prolonging drugs are responsible for almost all life-threatening "B-blocker failures" in LOT1.4)27) Thus, the differentiation between " $\beta$-blocker failure" and "patient failure" is important in the management of symptomatic patients after B-blocker treatment. B-blockers are more effective in LOT1 than in LOT2 or LOT3. 2)4)28)

B-blockers are very effective in preventing exercise-triggered cardiac events, but they have no significant effect on arousal- or sleep/rest-triggered cardiac events in LOT1 or LQT2 patients. ${ }^{29) 30)}$ However, B-blockers should be administered to patients with nonexercise related cardiac events since subsequent exercise-triggered events may still occur in this population. ${ }^{29)}$

B-blockers ingested by a mother are transmitted to the nursing infant through milk. As B-blocker is effective in preventing cardiac events in the postpartum period, B-blocker medication should be continued during this period as the benefit to the mother far outweighs the negligible risk to the nursing infant. ${ }^{14)}$

B-blocker-induced symptomatic bradycardia is an extremely rare event if the dosage is gradually increased. Side effects of B-blockers are loss of prowess, fatigue, weight gain, depression, and 
aggravation of asthma. ${ }^{27)}$ However, bronchial asthma is no longer considered an absolute contraindication to B-blockers. ${ }^{311}$ B-blockers are also associated with the development of clinically important hypoglycemia in young children, especially those with LQT2. ${ }^{32 / 33)}$

Long acting B-blockers in maximally tolerated doses are recommended and abrupt discontinuation should be avoided. Initially, all B-blockers were considered to be equally effective. ${ }^{24)}$ Recently, some experts suggested that not all B-blockers are equally effective in preventing cardiac events, although clear evidences supporting this issue seem to be limited. They believe that propranolol (2-5 mg/kg/day) and nadolol (1-2 $\mathrm{mg} / \mathrm{day} / \mathrm{kg}$ ) are definitely more effective than metoprolol, and probably atenolol. ${ }^{34-37)}$ However, another large study $(n=1530)$ showed that atenolol, metoprolol, propranolol, and nadolol were equally effective in reducing the risk of a first cardiac event in LQTS. ${ }^{38}$ Their efficacy differed with the genotype; nadolol was the only B-blocker associated with a significant risk reduction in LOT2 and propranolol was the least effective drug in the high-risk group of patients who experienced cardiac events during B-blocker therapy. ${ }^{38)}$ The cause of this discrepancy is not clear. Probably the differences in clinical characteristics of the patients and both dosage and dosing intervals of each B-blocker may have caused this discrepancy. Nadolol is not available in many countries including Korea.

\section{Left Cardiac Sympathetic Denervation}

LCSD is a rarely performed procedure but it is quite effective. ${ }^{26)}$ It has been described as having "near ICD levels of protection with less comorbidity". ${ }^{99)}$ LCSD's anti-fibrillatory effect is greatest in LOT1, followed by LOT2 and LOT3. ${ }^{40 / 411} \mathrm{Dr}$. Ackerman ${ }^{377}$ mentioned that none of his patients with single LOT1 mutations experienced a cardiac event after undergoing LCSD and the comorbidities associated with LCSD are much less than those related to ICD implantation. LCSD plays an important role in the proper management of LQTS patients as it could reduce the huge gap between a relatively simple B-blocker medication only and additional ICD implantation. This procedure reduces norepinephrine release at the ventricular level and raises the threshold for ventricular fibrillation without any reduction in cardiac contractility or heart rate. ${ }^{42 / 43)}$

Nowadays, LCSD is usually performed in very-high risk infants and small children to serve as a "bridge to an ICD." The usual procedure is high thoracic left sympathectomy and involves ablation of the lower half of the stellate ganglion along with $\mathrm{T} 2$ to T4. The results of LCSD are heavily influenced by the experience of the surgeon, as complete resection of the lower half of the left stellate ganglion is critical to the antifibrillatory effects of LCSD. ${ }^{39441)}$

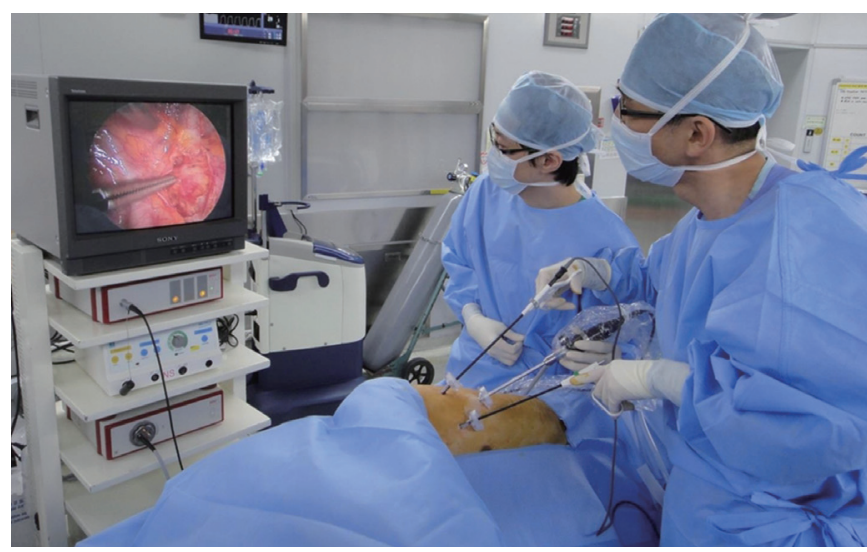

Fig. 2. Left cardiac sympathetic denervation is performed as a videoassisted thoracoscopic surgery.

The largest study on LCSD ( $n=147)$ showed a 91\% reduction in cardiac events per patient during a mean follow-up of 8 years. ${ }^{411}$ The majority of the patients were at high risk of cardiac events with an extremely prolonged QT interval (mean QTc $543 \pm 65 \mathrm{~ms}$ ). The recently introduced videoscopic LCSD is associated with a short hospital stay and less morbidity (Fig. 2). This procedure is quite similar to sympathectomy for hyperhidrosis, and most Korean chest surgeons are familiar with sympathectomy. The surgical time is usually less than 1 hour, and the patient can be discharged within a few days in experienced centers.

Whenever cardiac events recur in patients on B-blockers, LCSD should be strongly considered. The current indications are as follows: 1) patients with (frequent) appropriate ICD shocks, 2) patients with cardiac events during adequate B-blocker therapy, 3) B-blocker intolerant patients, and 4) very young and/or small patients who are considered technically inappropriate for ICD implantation. ${ }^{26 / 41}$ LCSD is especially effective in patients with B-blocker noncompliance and intolerance. ${ }^{40 / 411}$ Prophylactic LCSD in selected patients may improve the quality of life resulting from medication-related side effects. ${ }^{40)}$ Unfortunately, approximately 50\% of high risk patients have experienced more than one cardiac event after LCSD. ${ }^{40 / 411}$ Hence, LCSD must not be viewed as curative or as an ICD-alternative for high risk patients. The most common side effects of LCSD are dry left arm and face and profuse sweating on the right side. ${ }^{44)}$ Complications such as Horner's syndrome or droopy eyelid is very rare and mostly transient. ${ }^{411} \mathrm{~A}$ recently published study showed that most of the patients or their parents were satisfied with LCSD and would recommend LCSD to another patient. ${ }^{44)}$

\section{ICD Implantation}

The usual indications of ICD implantation are patients who have 
survived a cardiac arrest, patients with syncope despite adequate B-blocker therapy (and LCSD), and some patients considered as high risk with a very long QTc interval ( $>550 \mathrm{~ms})$, or signs of electrical instability such as T-wave alternans. $\left.{ }^{3 / 45}\right)$ The 2013 HRS/EHRA/APHRS guidelines do not recommend ICD implantation in asymptomatic LOTS patients who have not been tried on B-blocker therapy except under "special circumstances"; 1) OTc $>550$ ms in a patient other than LOT1; 2) LOT2 women with QTc >500 ms; 3) Jervell and LangeNielsen syndrome diagnosis; 4) strong family history of LQTS. ${ }^{3)}$ The risk for appropriate shocks in primary prevention patients with LQTS approached zero. ${ }^{46)}$ The current guidelines do not recommend ICD implantation in patients with acquired LOTS. However, it may also be beneficial in acquired LOTS patients as one study showed that $44 \%$ of patients with acquired LOTS and ICD received appropriate ICD shocks during a 7-year follow-up. ${ }^{47)}$

ICD implantation in young and active patients usually leads to inevitable complications such as inappropriate shocks, lead problems, vascular occlusion, infection, psychological adjustment, and social discrimination. ${ }^{48 / 49)}$ However, the diagnosis of a symptomatic LOTS, especially LOT3, usually leads to an ICD implantation. ${ }^{2 / 35)} \mathrm{A}$ recent European study showed that "Drugs and ICD implantation" was the first-line treatment of LOTS in 19\% of participating centers. ${ }^{26)}$ Recommending ICD implantation in a symptomatic LOTS patient is much easier than not recommending implantation of an ICD in this era of "defensive medicine." The ICD implantation rate seems to be as high as 75\% in some centers..$^{50)}$ One recent study with 157 patients showed a trend that the majority of patients had Class II and Class III indications for ICD implantation. ${ }^{48)}$ Although the ICD implantation frequency was highest among LOT3 patients, the greatest "save" rate occurred among LOT2 women, who were assessed to be at high risk. ${ }^{50)}$

The largest study on ICD ( $n=233$, mean age at implantation $=30 \pm 17$ years) showed that female (77\%) and LQT3 (22\% of known genotype) patients had a disproportionately high probability of being implanted with an ICD. ${ }^{45}$ ) During a follow-up of $4.6 \pm 3.2$ years, at least 1 appropriate shock was received by $28 \%$ of the patients, and adverse events occurred in 25\% of the patients. Interestingly, $>50 \%$ of their patients had not suffered a cardiac arrest and many had not even failed B-blocker therapy. Some patients were even asymptomatic. For proper selection of patients for ICD implantation, a new scoring system based on readily available simple clinical variables has been suggested. ${ }^{45}$ Studies performed at the Mayo clinic clearly showed that the majority of LOTS patients could be managed effectively without ICD implantation, and LQTS-triggered death did not occur in the $>500$ LOTS patients managed without an ICD. ${ }^{3750)}$ Recently, some experts cautiously suggested that patients who survived a cardiac arrest without previous B-blocker therapy can be managed without ICD implantation, especially LQT1 patients. They think that B-blockers and LCSD may suffice in such cases. As dual-chamber ICD was not effective in reducing the rate of inappropriate shocks, the decision to implant a dual-chamber ICD without a pacing indication should be avoided to reduce lead related complications. ${ }^{49)}$ To prevent inappropriate shocks, thoughtful programming is necessary, and it usually requires a ventricular fibrillation only zone, with a cutoff rate $>220-240$ bpm. $^{3}$

\section{Gene-Specific Therapy}

Potassium supplementation will increase serum potassium levels and may partially correct the repolarization abnormality in patients with LOT2. Two small studies showed that potassium supplementation with spironolactone resulted in significant OTC shortening. ${ }^{51152)}$ However, the long-term safety and efficacy is not known considering the risk of hyperkalemia and gynecomastia.

Sodium channel blockers such as mexiletine, flecainide, and ranolazine have been used to a limited extent in high risk LOT3 patients refractory to B-blockers or in patients with recurrent events despite ICD and LCSD therapies. ${ }^{233}$ However, their use is limited due to the following reasons: the response is not consistent and mutation specific, ECG response may not correlate with clinical efficacy, and long-term data are not available.

\section{Conclusion}

Life-style modification, B-blockers, LCSD, and ICD implantation are important therapeutic modalities in proper management of patients with LQTS. Prudent consideration is needed before making a decision to recommend an ICD implantation in a young and otherwise active patient. LCSD is still severely underutilized.

\section{Acknowledgements}

I thank SungHee Kim, M.D. for her assistance in the preparation of this manuscript.

\section{References}

1. Moss AJ, Schwartz PJ, Crampton RS, et al. The long QT syndrome. Prospective longitudinal study of 328 families. Circulation $1991 ; 84: 1136-44$ 
2. Lee $\mathrm{YS}$, Kwon BS, Kim GB, et al. Long OT syndrome: a Korean single center study. J Korean Med Sci 2013;28:1454-60.

3. Priori SG, Wilde AA, Horie M, et al. HRS/EHRA/APHRS expert consensus statement on the diagnosis and management of patients with inherited primary arrhythmia syndromes. Heart Rhythm 2013;10:1932-63.

4. Schwartz PJ, Priori SG, Spazzolini C, et al. Genotype-phenotype correlation in the long-OT syndrome: gene-specific triggers for lifethreatening arrhythmias. Circulation 2001;103:89-95.

5. Choi G, Kopplin $\sqcup$, Tester DJ, Will ML, Haglund CM, Ackerman MJ. Spectrum and frequency of cardiac channel defects in swimmingtriggered arrhythmia syndromes. Circulation 2004;110:2119-24.

6. Zipes DP, Ackerman MJ, Estes NA 3rd, Grant AO, Myerburg RJ, Van Hare G. Task Force 7: arrhythmias. J Am Coll Cardiol 2005;45:1354-63.

7. Pelliccia A, Fagard R, Bjørnstad HH, et al. Recommendations for competitive sports participation in athletes with cardiovascular disease: a consensus document from the Study Group of Sports Cardiology of the Working Group of Cardiac Rehabilitation and Exercise Physiology and the Working Group of Myocardial and Pericardial Diseases of the European Society of Cardiology. Eur Heart J 2005;26:1422-45.

8. Johnson JN, Ackerman MJ. Return to play? Athletes with congenital long QT syndrome. Br J Sports Med 2013;47:28-33.

9. Ackerman MJ, Zipes DP, Kovacs RJ, Maron BJ. Eligibility and Disqualification Recommendations for Competitive Athletes With Cardiovascular Abnormalities: Task Force 10: The Cardiac Channelopathies: A Scientific Statement From the American Heart Association and American College of Cardiology. J Am Coll Cardiol 2015;66:2424-8.

10. Lampert $R$, Olshansky $B$, Heidbuchel $H$, et al. Safety of sports for athletes with implantable cardioverter-defibrillators: results of a prospective, multinational registry. Circulation 2013;127:2021-30.

11. Christian S, Somerville M, Taylor S, Atallah J. Exercise and $\beta$-blocker therapy recommendations for inherited arrhythmogenic conditions. Cardiol Young 2016;26:1123-9.

12. Loar RW, Bos JM, Cannon BC, Ackerman MJ. Sudden cardiac arrest during sex in patients with either catecholaminergic polymorphic ventricular tachycardia or long-OT syndrome: a rare but shocking experience. J Cardiovasc Electrophysio/ 2015;26:300-4.

13. Wilde AA, Jongbloed RJ, Doevendans PA, et al. Auditory stimuli as a trigger for arrhythmic events differentiate HERG-related (LOTS2) patients from KVLOT1-related patients (LOTS1). J Am Coll Cardiol 1999;33:327-32.

14. Seth $R$, Moss AJ, McNitt $S$, et al. Long QT syndrome and pregnancy. J Am Coll Cardio/ 2007;49:1092-8.

15. Schwartz PJ, Ackerman MJ, George AL Jr, Wilde AA. Impact of genetics on the clinical management of channelopathies. J Am Coll
Cardio/ 2013;62:169-80.

16. Rosero SZ, Zareba W, Moss AJ, et al. Asthma and the risk of cardiac events in the Long QT syndrome Investigative Group. Am J Cardiol 1999;84:1406-11.

17. Thottathil P, Acharya J, Moss AJ, et al. Risk of cardiac events in patients with asthma and long-OT syndrome treated with beta2 agonists. Am J Cardio/ 2008;102:871-4.

18. Mall M, Wissner $A$, Schreiber $R$, et al. Role of $K(V) L O T 1$ in cyclic adenosine monophosphate-mediated $\mathrm{Cl}(-)$ secretion in human airway epithelia. Am J Respir Cell Mol Biol 2000;23:283-9.

19. Collins S, Widger J, Davis A, Massie J. Management of asthma in children with long OT syndrome. Paediatr Respir Rev 2012;13:100-5.

20. Crean AM, Abdel-Rahman SE, Greenwood JP. A sweet tooth as the root cause of cardiac arrest. Can J Cardio/ 2009;25:e357-8.

21. Lin C, Ke X, Ranade V, Somberg J. The additive effects of the active component of grapefruit juice (naringenin) and antiarrhythmic drugs on HERG inhibition. Cardiology 2008;110:145-52.

22. Abu-Zeitone A, Peterson DR, Polonsky B, McNitt S, Moss AJ. Oral contraceptive use and the risk of cardiac events in patients with long OT syndrome. Heart Rhythm 2014;11:1170-5.

23. Zhang C, Kutyifa V, Moss AJ, McNitt S, Zareba W, Kaufman ES. LongOT syndrome and therapy for attention deficit/hyperactivity disorder J Cardiovasc Electrophysio/ 2015;26:1039-44.

24. Moss AJ, Zareba W, Hall WJ, et al. Effectiveness and limitations of beta-blocker therapy in congenital long-OT syndrome. Circulation 2000;101:616-23.

25. Park YM, Kim SJ, Park CH, et al. Repeated aborted sudden cardiac death with long QT syndrome in a patient with anomalous origin of the right coronary artery from the left coronary cusp. Korean Circ $J$ 2013:43:830-3.

26. Hocini M, Pison L, Proclemer $A_{1}$ et al. Diagnosis and management of patients with inherited arrhythmia syndromes in Europe: results of the European Heart Rhythm Association Survey. Europace 2014;16:600-3.

27. Vincent GM, Schwartz PJ, Denjoy I, et al. High efficacy of betablockers in long-QT syndrome type 1: contribution of noncompliance and QT-prolonging drugs to the occurrence of beta-blocker treatment "failures". Circulation 2009;119:215-21.

28. Priori SG, Napolitano C, Schwartz PJ, et al. Association of long QT syndrome loci and cardiac events among patients treated with betablockers. JAMA 2004;292:1341-4.

29. Goldenberg I, Thottathil P, Lopes CM, et al. Trigger-specific ionchannel mechanisms, risk factors, and response to therapy in type 1 long QT syndrome. Heart Rhythm 2012;9:49-56.

30. Kim JA, Lopes CM, Moss AJ, et al. Trigger-specific risk factors and response to therapy in long OT syndrome type 2. Heart Rhythm 2010;7:1797-805. 
31. Viskin S, Halkin A. Treating the long-QT syndrome in the era of implantable defibrillators. Circulation 2009;119:204-6.

32. Koponen $M$, Marjamaa A, Hiippala A, et al. Follow-up of 316 molecularly defined pediatric long-QT syndrome patients: clinical course, treatments, and side effects. Circ Arrhythm Electrophysiol 2015;8:815-23.

33. Poterucha JT, Bos JM, Cannon BC, Ackerman MJ. Frequency and severity of hypoglycemia in children with beta-blocker-treated long OT syndrome. Heart Rhythm 2015;12:1815-9.

34. Chockalingam $P$, Crotti L, Girardengo G, et al. Not all beta-blockers are equal in the management of long OT syndrome types 1 and 2: Higher recurrence of events under metoprolol. J Am Coll Cardiol 2012;60:2092-9.

35. Schwartz PJ, Ackerman MJ. The long OT syndrome: a transatlantic clinical approach to diagnosis and therapy. Eur Heart $J$ 2013;34:3109-16.

36. Schwartz PJ. My Approach to the long QT syndrome(LOTS). Trends Cardiovasc Med 2015;25:376-7.

37. Ackerman MJ. My Approach to treatment of the congenital long QT syndromes. Trends Cardiovasc Med 2015;25:67-9.

38. Abu-Zeitone A, Peterson DR, Polonsky B, McNitt S, Moss AJ. Efficacy of different beta-blockers in the treatment of long QT syndrome. $J$ Am Coll Cardio/ 2014;64:1352-8.

39. Schwartz PJ. Cutting nerves and saving lives. Heart Rhythm 2009;6:760-3.

40. Bos JM, Bos KM, Johnson JN, Moir C, Ackerman MJ. Left cardiac sympathetic denervation in long QT syndrome: analysis of therapeutic nonresponders. Circ Arrhythm Electrophysio/ 2013;6:705-11.

41. Schwartz PJ, Priori SG, Cerrone M, et al. Left cardiac sympathetic denervation in the management of high-risk patients affected by the long-QT syndrome. Circulation 2004;109:1826-33.

42. Schwartz PJ, Snebold NG, Brown AM. Effects of unilateral cardiac sympathetic denervation on the ventricular fibrillation threshold. Am J Cardio/ 1976;37:1034-40.

43. Schwartz PJ, Stone HL. Effects of unilateral stellectomy upon cardiac performance during exercise in dogs. Circ Res 1979;44:637-45.

44. Antiel RM, Bos JM, Joyce DD, et al. Quality of life after videoscopic left cardiac sympathetic denervation in patients with potentially lifethreatening cardiac channelopathies/cardiomyopathies. Heart Rhythm 2016;13:62-9.

45. Schwartz PJ, Spazzolini C, Priori SG, et al. Who are the long-QT syndrome patients who receive an implantable cardioverterdefibrillator and what happens to them?: data from the European Long-OT Syndrome Implantable Cardioverter-Defibrillator (LOTS ICD) Registry. Circulation 2010;122:1272-82.

46. Olde Nordkamp LR, Wilde AA, Tijssen JG, Knops RE, van Dessel PF, de Groot JR. The ICD for primary prevention in patients with inherited cardiac diseases: indications, use, and outcome: a comparison with secondary prevention. Circ Arrhythm Electrophysio/ 2013;6:91-100.

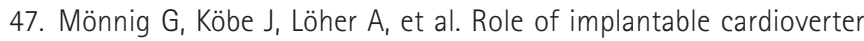
defibrillator therapy in patients with acquired long OT syndrome: a long-term follow-up. Europace 2012;14:396-401.

48. Gaba P, Bos JM, Cannon BC, et al. Implantable cardioverterdefibrillator explantation for overdiagnosed or overtreated congenital long QT syndrome. Heart Rhythm 2016;13:879-85.

49. Olde Nordkamp LR, Postema PG, Knops RE, et al. Implantable cardioverter-defibrillator harm in young patients with inherited arrhythmia syndromes: a systematic review and meta-analysis of inappropriate shocks and complications. Heart Rhythm 2016;13:443-54.

50. Horner JM, Kinoshita M, Webster TL, Haglund CM, Friedman PA, Ackerman MJ.,Implantable cardioverter defibrillator therapy for congenital long QT syndrome: a single-center experience. Heart Rhythm 2010;7:1616-22.

51. Compton SJ, Lux RL, Ramsey MR, et al. Genetically defined therapy of inherited long-QT syndrome. Correction of abnormal repolarization by potassium. Circulation 1996;94:1018-22.

52. Etheridge SP, Compton SJ, Tristani-Firouzi M, Mason JW. A new oral therapy for long QT syndrome: long-term oral potassium improves repolarization in patients with HERG mutations. J Am Coll Cardiol 2003;42:1777-82. 